\title{
Outage Performance of Flexible OFDM Schemes in Packet-Switched Transmissions
}

\author{
Romain Couillet ${ }^{1,2}$ and Mérouane Debbah ${ }^{2}$ \\ ${ }^{1}$ Algorithm Group, ST-Ericsson, 635 Route des Lucioles, 06560 Sophia-Antipolis, France \\ ${ }^{2}$ Department of Telecommunication, Alcatel Lucent Chair on Flexible Radio, Supélec, 3 rue Joliot Curie, 91192 Gif sur Yvette, France
}

Correspondence should be addressed to Romain Couillet, romain.couillet@gmail.com

Received 30 January 2009; Revised 18 June 2009; Accepted 7 August 2009

Recommended by Ananthram Swami

$\alpha$-OFDM, a generalization of the OFDM modulation, is proposed. This new modulation enhances the outage capacity performance of bursty communications. The $\alpha$-OFDM scheme is easily implementable as it only requires an additional time symbol rotation after the IDFT stage and a subsequent phase rotation of the cyclic prefix. The physical effect of the induced rotation is to slide the DFT window over the frequency spectrum. When successively used with different angles $\alpha$ at the symbol rate, $\alpha$-OFDM provides frequency diversity in block fading channels. Interestingly, simulation results show a substantial gain in terms of outage capacity and outage BER in comparison with classical OFDM modulation schemes. The framework is extended to multiantenna and multicellular OFDM-based standards. Practical simulations, in the context of 3GPP-LTE, called hereafter $\alpha$-LTE, sustain our theoretical claims.

Copyright () 2009 R. Couillet and M. Debbah. This is an open access article distributed under the Creative Commons Attribution License, which permits unrestricted use, distribution, and reproduction in any medium, provided the original work is properly cited.

\section{Introduction}

With the recent growth of wireless communications and the increasing demand for high transmission rates, orthogonal frequency division multiplexing (OFDM) is being considered the desirable modulation scheme of most future wireless communication technologies. Many wireless standards [13] have already rallied in favour of OFDM. The attractive features of OFDM are numerous; a key advantage over other classical modulation schemes is that OFDM can be designed to reach a high spectral efficiency. The main advantage in practice lies in the flat fading aspect of the channel that facilitates equalization at the receiver side. This property originates from the cyclic prefix $(\mathrm{CP})$ addition, prior to signal transmission, that allows to model the channel as a circulant matrix in the time-domain [4]. Those circulant matrices are diagonalizable in the Fourier basis, hence the seemingly flat fading aspect of the channel in the frequency domain. The analysis of circulant matrices, and their generalization, is the starting point of the present work.

In addition to the demand for high transmission rates, recent wireless standards have also steadily moved from the connected circuit-switched transmission mode to the bursty packet-switched transmission mode. The main drawback of the packet-switched mode arises when the transmission time is less than the channel coherence time [5], as the channel is then static over the communication duration. Indeed, for any target transmission rate $R$, there exists a nonnull probability that the channel is so ill conditioned that the resulting achievable rate is less than $R$. This outage probability is especially nonnegligible in OFDM when the channel delay spread is small, or equivalently when the channel coherence bandwidth [5] is large. These observations have led to consider methods which provide channel diversity to protect the transmitted symbols from deep channel fading. Among those methods, [6] proposed a dynamic beamforming scheme using multiple antennas, known as dumb antennas, which induces fast channel variations over time. The relevant effect of this method is to increase the channel diversity during the transmission period. Recently, [7] introduced a compact MIMO system which mimics the behaviour of multiple antennas from one single rotating antenna, thus producing additional degrees of freedom. A desirable method to cope with the outage problem in OFDM is to provide diversity in the frequency domain; this is accomplished by the cyclic delay diversity method [8] by 
introducing different symbol delays on an array of transmit antennas. However, all those methods require additional antennas to provide channel diversity or are still difficult to implement in practice.

In the following work, we first introduce a new single antenna modulation scheme called $\alpha$-OFDM, which produces frequency diversity with neither a tremendous increase in complexity nor additional antenna requirement. The main idea is to successively transmit data on different sets of frequency carriers, with no need for a higher layer frequency allocation scheduler. In particular, we show that $\alpha$-OFDM allows to flexibly reuse adjacent frequency bands by properly adjusting a single (rotation) parameter: $\alpha$.

This work is then extended to the multiuser OFDMA (multiple access OFDM) case, where 3GPP-Long Term Evolution (LTE) is used as benchmark for comparison against classical OFDM. Although both $\alpha$-LTE and LTE with frequency hopping techniques seem to be similar, the $\alpha$-OFDM algorithm is more flexible and can be adapted on a per-OFDM symbol rate without advanced scheduling methods. $\alpha$-OFDM can also be seen as yet another technique applicable for software-defined radios $[9,10]$ for it enables flexible frequency management by means of digital processing.

The rest of the paper unfolds as follows: in Section 2, we study the mathematical extension of circulant matrices, which is at the heart of the classical OFDM modulation and we introduce the novel $\alpha$-OFDM scheme. Practical communication systems, based on $\alpha$-OFDM, are then introduced in Section 3 and are shown to benefit from the frequency diversity offered by $\alpha$-OFDM in terms of outage capacity. The theoretical claims are validated by simulations in Section 4 . We then introduce in Section 5 some practical applications and quantify the outage performance gain. In particular we propose an extension for the 3GPP LTE standard, called hereafter $\alpha$-LTE, which is further extended into a general $\alpha$-OFDMA scheme. Finally, conclusions are provided in Section 6.

Notations. In the following, boldface lower and capital case symbols represent vectors and matrices, respectively. The transposition is denoted $(\cdot)^{\top}$ and the Hermitian transpose is $(\cdot)^{\mathrm{H}}$. The operator $\operatorname{diag}(\mathbf{x})$ turns the vector $\mathbf{x}$ into a diagonal matrix. The symbol $\operatorname{det}(\mathbf{X})$ is the determinant of matrix $\mathbf{X}$. The symbol E[·] denotes expectation. The binary relation symbol $X \mid Y$ means that $Y$ is divisible by $X$.

\section{System Model}

The basic idea of this work relies on a mathematical generalization of the diagonalization property of circulant matrices. More specifically we introduce in the following the so-called $(\rho, \alpha)$-circulant matrices which can be diagonalized in a modified Fourier basis, hereafter referred to as the $(\rho, \alpha)$ Fourier basis. This will entail the introduction of the $\alpha$ OFDM scheme, which in turn generalizes the OFDM concept and whose key physical property is to provide dynamic subcarrier frequency shift controlled by the parameter $\alpha$.
2.1. Mathematical Preliminaries. Let us first recall the diagonalization property of circulant matrices.

Definition 1. A circulant matrix $\mathbf{H}_{0}$ of size $N$ with basevector $\mathbf{h}=\left\{h_{0}, \ldots, h_{L-1}\right\} \in \mathbb{C}^{L}(L \leq N)$ is the $N \times N$ Toeplitz matrix:

$$
\mathbf{H}_{0}=\left[\begin{array}{ccccccc}
h_{0} & 0 & \cdots & 0 & h_{L-1} & \cdots & h_{1} \\
h_{1} & h_{0} & \ddots & & \ddots & \ddots & \vdots \\
\vdots & & \ddots & \ddots & & \ddots & h_{L-1} \\
h_{L-1} & & & \ddots & \ddots & & 0 \\
0 & \ddots & & & \ddots & \ddots & \vdots \\
\vdots & \ddots & \ddots & & & h_{0} & 0 \\
0 & \cdots & 0 & h_{L-1} & \cdots & h_{1} & h_{0}
\end{array}\right] .
$$

Circulant matrices can be diagonalized by $\mathbf{F}_{N}$, the discrete Fourier transform (DFT) matrix of size $N$. The eigenvalues in their spectral decomposition are formed by the DFT of their first column $\left[h_{0}, \ldots, h_{L-1}, 0, \ldots, 0\right]^{\top}[11]$.

Those circulant matrices actually enter a broader class of matrices, which we will call $(\rho, \alpha)$-circulant matrices. Those are defined as follows.

Definition 2. For $z=\rho e^{i \alpha} \in \mathbb{C},(\rho, \alpha) \in \mathbb{R}^{+} \times \mathbb{R}$, we call an $N \times N$ matrix $\mathbf{H}_{(\rho, \alpha)}(\rho, \alpha)$-circulant if it is of the form

$$
\mathbf{H}_{(\rho, \alpha)}=\left[\begin{array}{ccccccc}
h_{0} & 0 & \cdots & 0 & \rho e^{i \alpha} h_{L-1} & \cdots & \rho e^{i \alpha} h_{1} \\
h_{1} & h_{0} & \ddots & & \ddots & \ddots & \vdots \\
\vdots & & \ddots & \ddots & & \ddots & \rho e^{i \alpha} h_{L-1} \\
h_{L-1} & & & \ddots & \ddots & & 0 \\
0 & \ddots & & & \ddots & \ddots & \vdots \\
\vdots & \ddots & \ddots & & & h_{0} & 0 \\
0 & \cdots & 0 & h_{L-1} & \cdots & h_{1} & h_{0}
\end{array}\right] .
$$

This is a matrix with first column $\left[h_{0}, \ldots, h_{L-1}, 0, \ldots, 0\right]^{\top}$, and subsequent columns successive cyclic shifts of this column. The upper triangular part of the matrix is multiplied by $\rho e^{i \alpha}$.

Proposition 1. All $N \times N(\rho, \alpha)$-circulant matrices are diagonalizable by the $(\rho, \alpha)$-Fourier matrix $\mathbf{F}_{N,(\rho, \alpha)}$, defined as

$$
\mathbf{F}_{N,(\rho, \alpha)}=\mathbf{F}_{N} \cdot \operatorname{diag}\left(1, \rho^{(1 / N)} e^{i \alpha(1 / N)}, \ldots, \rho^{(N-1) / N} e^{i \alpha((N-1) / N)}\right)
$$




\section{Hence one denotes}

$$
\operatorname{diag}\left(H_{\alpha}(0), \ldots, H_{\alpha}(N-1)\right)=\mathbf{F}_{N,(\rho, \alpha)} \mathbf{H}_{(\rho, \alpha)} \mathbf{F}_{N,(\rho, \alpha)}^{-1},
$$

where the diagonal elements are given by the $(\rho, \alpha)$-DFT of the first column of $\mathbf{H}_{(\rho, \alpha)}$ :

$$
\left[H_{\alpha}(0), \ldots, H_{\alpha}(N-1)\right]^{\top}=\mathbf{F}_{N,(\rho, \alpha)}\left[h_{0}, \ldots, h_{L-1}, 0, \ldots, 0\right]^{\top} .
$$

Proof. The proof is an adaption of a proof from Gray [11], Section 3.1, where the author characterizes the eigenvectors and eigenvalues of a circulant matrix. Given a $(\rho, \alpha)$-circulant matrix $\mathbf{H}$, the eigenvalues $H_{\alpha}(m)$ and the eigenvectors $\mathbf{v}_{m}$ of $\mathbf{H}$ are the solutions of

$$
\mathbf{H v}=\phi \mathbf{v}
$$

This can be written in scalar form as the system of equations, for $m=0, \ldots, N-1$,

$$
\sum_{k=0}^{\min (m, L-1)} h_{k} \mathbf{v}_{m-k}+\rho e^{i \alpha} \sum_{k=m+1}^{L-1} h_{k} \mathbf{v}_{N-(k-m)}=\phi \mathbf{v}_{m} .
$$

In (7), we use the convention that the second summation is zero if $L-1<m+1$.

Let us assume $\mathbf{v}_{k}=\varrho^{k}$ and replace it in (7). Cancellation of $\varrho^{m}$ yields

$$
\sum_{k=0}^{\min (m, L-1)} h_{k} \varrho^{-k}+\rho e^{i \alpha} \varrho^{N} \sum_{k=m+1}^{L-1} h_{k} \varrho^{-k}=\phi .
$$

Thus, choosing $\varrho$ such that $\rho e^{i \alpha} \varrho^{N}=1$, we obtain the eigenvalue

$$
\phi=\sum_{k=0}^{L-1} k \varrho^{-k}
$$

and the associated (normalized) eigenvector

$$
\mathbf{v}=\beta\left[1, \varrho, \varrho^{2}, \ldots, \varrho^{N-1}\right]^{\top}
$$

with $\beta=1 / \sqrt{N}$ if $\rho=1$ and $\beta=\sqrt{\left(1-1 / \alpha^{1 / N}\right) /(1-1 / \alpha)}$ otherwise. Then we choose $\varrho_{m}$ as the complex $N^{\text {th }}$ root of $\rho e^{-i \alpha}, \varrho_{m}=1 / \rho^{1 / N} e^{-i \alpha / N} e^{2 \pi i m / N}$, obtaining the eigenvalue

$$
H_{\alpha}(m)=\sum_{j=0}^{L-1} h_{j} \rho^{j / N} e^{i \alpha(j / N)} e^{-2 \pi i j(m / N)}
$$

and eigenvector

$$
\mathbf{v}_{m}=\beta\left[1, \frac{e^{-i \alpha(1 / N)}}{\rho^{1 / N}} e^{2 \pi i(m / N)}, \ldots, \frac{e^{-i \alpha((N-1) / N)}}{\rho^{(N-1) / N}} e^{2 \pi i m((N-1) / N)}\right]^{\top}
$$

such that

$$
\mathbf{H v}_{m}=H_{\alpha}(m) \mathbf{v}_{m}, \quad m=0, \ldots, N-1 .
$$

From (11), we can deduce immediately an inverse transform (analog to the inverse Fourier transform) to obtain the elements of the first column of $\mathbf{H}$ from the eigenvalues

$$
\ell=\frac{1}{\rho^{\ell / N}} e^{-i \alpha(\ell / N)} \frac{1}{N} \sum_{m=0}^{N-1} H_{\alpha}(m) e^{2 \pi i m(\ell / N)}, \quad \ell=0, \ldots, L-1 .
$$

Therefore, $(\rho, \alpha)$-circulant matrices only differ from circulant matrices by (i) an additional rotation matrix turning the discrete Fourier transform matrix $\mathbf{F}_{N}$ into a $(\rho, \alpha)$ Fourier matrix $\mathbf{F}_{N,(\rho, \alpha)}$ and (ii) an additional rotation of the upper-triangular part of the circulant matrix. When applied to the OFDM communication chain (both at the transmit and receive sides), these alterations entail interesting physical properties which we introduce in the following.

2.2. OFDM. Let us first recall the classical OFDM modulation scheme and introduce our notations, before we present the more general $\alpha$-OFDM framework. Consider a regular OFDM transmission scheme. Denote by $\mathbf{s} \in \mathbb{C}^{N}$ the transmitted OFDM symbol, $\mathbf{n} \in \mathbb{C}^{N}$ some additive white Gaussian noise (AWGN) sensed by the receiver with entries of variance $\mathrm{E}\left[\left|n_{i}\right|^{2}\right]=\sigma^{2}$, and $\mathbf{H}_{0}$ the circulant time-domain channel matrix, as in (1). The time-domain received signal $\mathbf{r} \in \mathbb{C}^{N}$ reads

$$
\mathbf{r}=\mathbf{H}_{0} \mathbf{F}^{\mathrm{H}} \mathbf{s}+\mathbf{n},
$$

where $\mathbf{F}_{N}$ is rewritten $\mathbf{F}$ for the sake of readability. Therefore $\mathbf{H}_{0}$ is diagonalizable by the Fourier matrix $\mathbf{F}$, with diagonal elements being the discrete Fourier transform of the first column $\left[h_{0}, \ldots, h_{L-1}, 0, \ldots, 0\right]^{\top}$. This is simply obtained by multiplying $\mathbf{r}$ in (15) by $\mathbf{F}$. The distribution of the noise does not change, since a unitary transformation of a Gaussian i.i.d. (independent and identically distributed) vector is still a Gaussian vector of same mean and variance. Thus,

$$
\mathbf{F} \cdot \mathbf{r}=\operatorname{diag}\left(H_{0}(0), \ldots, H_{0}(N-1)\right) \mathbf{s}+\mathbf{n}
$$

with $H_{0}(\cdot)$ being the DFT of the first column of $\mathbf{H}_{0}$ :

$$
H_{0}(m)=\sum_{j=0}^{L-1} j e^{-2 \pi i j(m / N)} .
$$

2.3. $\alpha$-OFDM. Due to the mathematical generalization of circulant matrices previously proposed, the classical OFDM scheme can in turn be generalized in form of the $\alpha$-OFDM scheme which we define as follows. At the transmitter side, the $\alpha$-OFDM scheme is similar to the classical OFDM modulation, except that it requires

(i) the frequency-domain OFDM signal vector $s$ to be first multiplied by the matrix

$$
\operatorname{diag}\left(1, e^{-i \alpha / N}, \ldots, e^{-i \alpha(N-1) / N}\right)
$$

after the inverse DFT (IDFT) stage in the OFDM transmission chain, 


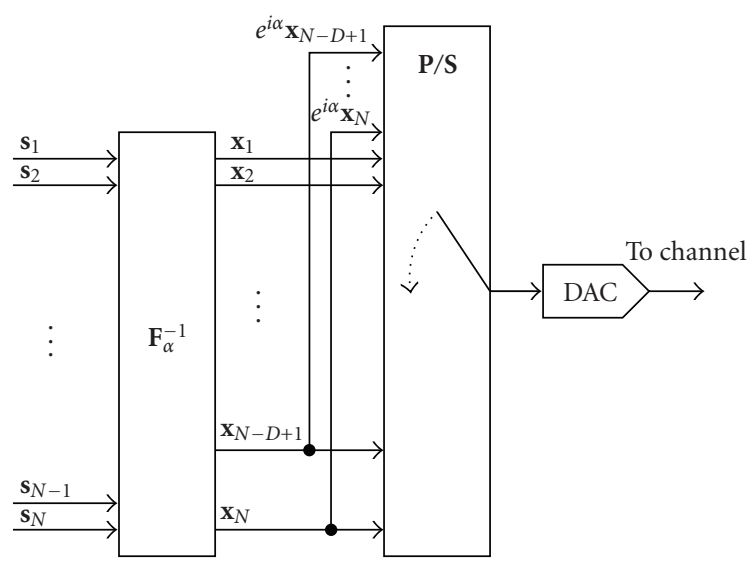

FIGURE 1: $\alpha$-OFDM transmission scheme.

(ii) the time-domain symbols of the CP to be multiplied by the constant $z=\rho e^{i \alpha}$ where we set here $\rho=1$.

Note that if $\rho$ were chosen different from $1, \mathbf{F}_{N,(\rho, \alpha)}$ would not be unitary, which would generate noise amplification at the receiver side.

Hence, the time-domain received signal $\mathbf{r}$ reads

$$
\mathbf{r}=\mathbf{H}_{\alpha} \mathbf{F}_{\alpha}^{\mathrm{H}} \mathbf{s}+\mathbf{n}
$$

where $\mathbf{F}_{\alpha}$ is a simplified notation for $\mathbf{F}_{N,(1, \alpha)}$, and $\mathbf{H}_{\alpha}$ is the transmission channel matrix. The $\alpha$-OFDM transmission chain is depicted in Figure 1. Considering the mathematical development previously presented, the channel matrix $\mathbf{H}_{\alpha}$ associated to this model is $(1, \alpha)$-circulant (for short, this will now be referred to as $\alpha$-circulant) and therefore diagonalizable in the $\alpha$-Fourier domain.

At the receiver side, the $\mathrm{CP}$ is discarded like in the classical OFDM scheme and the receive useful data are multiplied by the matrix $\mathbf{F}_{\alpha}$. Mathematically, this is

$$
\mathbf{F}_{\alpha} \cdot \mathbf{r}=\operatorname{diag}\left(H_{\alpha}(0), \ldots, H_{\alpha}(N-1)\right) \mathbf{s}+\mathbf{n}
$$

with $H_{\alpha}(\cdot)$ being the $\alpha$-DFT of the first column of $\mathbf{H}_{\alpha}$ :

$$
\begin{aligned}
H_{\alpha}(m) & =\sum_{j=0}^{L-1} h_{j} e^{-2 \pi i(j / N)(m-\alpha / 2 \pi)} \\
& \simeq H_{0}\left(\left\{\left\lceil m-\frac{\alpha}{2 \pi}\right\rceil\right\}_{N}\right)
\end{aligned}
$$

where $\{\cdot\}_{N}$ denotes the modulo $N$ operation, and $\lceil x\rceil$ denotes the closest integer larger than $x$. The approximation (22) deserves some more comments: classical OFDM systems are designed such that the typical channel coherence bandwidth is greater or equal to one subcarrier spacing. Therefore, the channel frequency gain decimal at decimal frequency $\{m-\alpha / 2 \pi\}_{N}$ is usually well approximated by that at frequency $\left\lceil\{m-\alpha / 2 \pi\}_{N}\right\rceil$.

Remark 1. Note that $H_{\alpha}(m)$ is a frequency-shifted version of $H_{0}(m)$. Thus $\alpha$-OFDM introduces a fractional frequency shift $\alpha / 2 \pi$ to the channel $\left\{H_{0}(0), \ldots, H_{0}(N-1)\right\}$ as shown

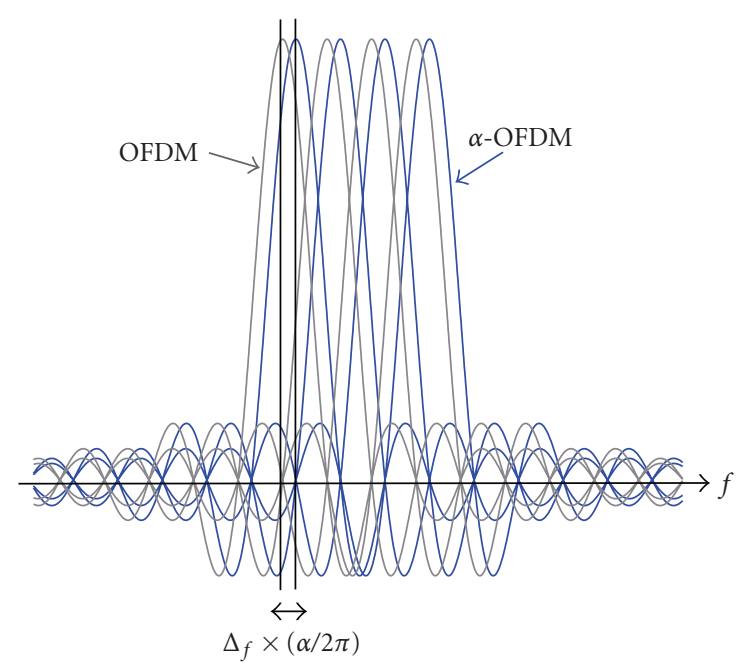

Figure 2: $\alpha$-OFDM.

in Figure 2. Particularly, if $\alpha / 2 \pi$ is an integer, then $\alpha$-OFDM merely remaps the OFDM symbol onto a circular-shifted version of the subcarriers.

Therefore $\alpha$-OFDM introduces only a minor change compared to OFDM and has for main incidence to circularly shift the OFDM subcarriers by a decimal value $\alpha / 2 \pi, \alpha \in \mathbb{R}$. Note that the classical OFDM modulation is the particular $\alpha$ OFDM scheme for which $\alpha=0$. By controlling the rotation angle $\alpha$ and allowing the use of adjacent frequency bands, $\alpha$-OFDM enables a computationally inexpensive frequency diversity, which in turn is known to increase outage capacity performance. The main results are introduced in the following.

\section{Outage Capacity Analysis}

The transmission rates achievable in bursty communications cannot be evaluated with Shannon's ergodic capacity which involves infinite delay transmission. Instead, the transmission capabilities of a bursty system are usually measured through the rate achievable $(100-q) \%$ of the time. This rate $C_{0}$ is known as the $q \%$-outage capacity and verifies $\mathbb{P}\left(C>C_{0}\right)=(100-q) / 100$, with $C$ being Shannon's capacity [12] for fixed channels.

3.1. $\alpha$-OFDM Capacity. The normalized, that is, per subcarrier, capacity $C$ of a regular OFDM system (also called spectral efficiency) for the fixed frequency-domain channel $\left\{H_{0}(0), \ldots, H_{0}(N-1)\right\}$ reads

$$
C=\frac{1}{N} \sum_{m=0}^{N-1} \log \left(1+\frac{\left|H_{0}(m)\right|^{2}}{\sigma^{2}}\right),
$$

while the capacity for $\alpha$-OFDM is

$$
C_{\alpha}=\frac{1}{N} \sum_{m=0}^{N-1} \log \left(1+\frac{\left|H_{\alpha}(m)\right|^{2}}{\sigma^{2}}\right) .
$$


In the rest of this document, we refer to capacity as the normalized system capacity. The system-wide capacity will be referred to as total capacity.

Remind that the channel coherence bandwidth of an OFDM system is at least as large as the subcarrier spacing (otherwise the channel delay spread would be longer than the OFDM symbol duration). Therefore, as already mentioned, $H_{\alpha}(m) \simeq H_{0}\left(\{\lceil m-\alpha / 2 \pi\rceil\}_{N}\right)$. From the expressions (23) and (24), we then conclude that $C \simeq C_{\alpha}$ in some sense. As a consequence, $\alpha$-OFDM does not bring any gain, in terms of either ergodic or outage capacity in this simple setting.

Nonetheless, for various reasons, such as the introduction of frequency guard bands or oversampling at the receiver, many OFDM systems with a size- $N$ DFT use a limited number $N_{u}<N$ of contiguous subcarriers to transmit useful data. Those are referred to as useful subcarriers. In such schemes, the OFDM capacity for the fixed channel $\mathbf{H}_{0}$ trivially extends to

$$
C=\frac{1}{N_{u}} \sum_{m=0}^{N_{u}-1} \log \left(1+\frac{\left|H_{0}(m)\right|^{2}}{\sigma^{2}}\right)
$$

while the equivalent for $\alpha$-OFDM is

$$
C_{\alpha}=\frac{1}{N_{u}} \sum_{m=0}^{N_{u}-1} \log \left(1+\frac{\left|H_{\alpha}(m)\right|^{2}}{\sigma^{2}}\right) .
$$

The sizes of the useful bandwidths of both OFDM and $\alpha$ OFDM are the same but their locations differ and therefore $C \neq C_{\alpha}$. However, using different frequency bands is only acceptable in practice if these bands are not protected, which is rarely the case in classical OFDM systems. In the subsequent sections, we will propose practical schemes to overcome this limitation at the expense of a small bandwidth sacrifice: that is, we will propose to discard subcarriers on the sides of the available bandwidth to allow the useful transmission band to "slide" over the total valid bandwidth using different values for $\alpha$. In the remainder of this paper, we therefore consider OFDM systems with $N$ subcarriers transmitting data over $N_{u}$ contiguous useful subcarriers whose locations are subject to different constraints.

\section{2. $\alpha$-OFDM-Based Systems}

3.2.1. $\alpha$-OFDM\#1. First consider a single-user OFDM system with the $N-N_{u}$ nonuseful subcarriers gathered into a contiguous subband, up to a circular rotation over the total bandwidth. This constraint allows to use $\alpha$-OFDM for any $\alpha \in \mathbb{R}$ as long as the $N_{u}$ useful subcarriers are gathered in a contiguous band. We then naturally introduce the $\alpha$ OFDM\#1 scheme as follows.

Let $M \in \mathbb{N}$ and $\mathcal{M}$ be a set of cardinality $M$ defined as

$$
\mathcal{M}=\left\{0,2 \pi \frac{N}{M}, \ldots, 2 \pi \frac{N(M-1)}{M}\right\},
$$

where $\alpha \in \mathbb{R}$ is a priori known both to the transmitter and to the receiver. The consecutive time-domain symbols are transmitted successively using a $(2 \pi k N / M)$-OFDM modulation, for $k$ ranging from 0 to $M-1$; that is. denoting $\mathbf{s}^{(k)}$ as the $k$ th transmitted symbol, $\mathbf{s}^{(1)}$ is sent using 0-OFDM, then $\mathbf{s}^{(2)}$ is sent using $(2 \pi N / M)$-OFDM and so on. At the receiver side, the corresponding $(2 \pi k N / M), k \in \mathbb{N}$, rotation values are used to align to the transmission band and to decode the received symbols. is

The persubcarrier capacity $C_{\# 1}$ of the $\alpha$-OFDM\#1 scheme

$$
C_{\# 1}=\frac{1}{M N_{u}} \sum_{m=0}^{N_{u}-1} \sum_{\alpha \in \mathcal{M}} \log \left(1+\frac{\left|H_{\alpha}(m)\right|^{2}}{\sigma^{2}}\right) .
$$

Note that the total capacity $C^{\left(W_{u}\right)}$ for a system of bandwidth $\mathcal{W}$ and useful bandwidth $\mathcal{W}_{u}=\mathcal{W} \cdot N_{u} / N$ scales with $N_{u}$, or equivalently with $\mathfrak{W}_{u}$ :

$$
C^{\left(W_{u}\right)}=W_{u} \cdot C_{\# 1}
$$

3.2.2. $\alpha$-OFDM\#2. Assuming perfect channel state information at the transmitter (CSIT), an improved scheme, $\alpha$ OFDM\#2, can be derived from $\alpha$-OFDM\#1, which selects among the $M$ values of $\mathcal{M}$ the one that maximizes the instantaneous capacity. Its capacity $C_{\# 2}$ reads

$$
C_{\# 2}=\frac{1}{N_{u}} \max _{\alpha \in \mathcal{M}} \sum_{m=0}^{N_{u}-1} \log \left(1+\frac{\left|H_{\alpha}(m)\right|^{2}}{\sigma^{2}}\right) .
$$

This scheme can prove useful when the channel coherence time is sufficiently long for the receiver to feed back channel information to the transmitter. The latter will then be allowed to transmit over one particular subchannel among the $M$ subchannels provided by the different bandwidth shifts. However, the transmitter must also inform the receiver of its choice of the $\alpha$ parameter; this can be performed by introducing an extra overhead to the first transmitted packet.

3.2.3. $\alpha$-OFDM\#3. Consider now that the protected unused subcarriers can only be placed on the lowest-frequency and highest-frequency sides of the bandwidth, as is the case of most practical systems. Following the same structure as the $\alpha$-OFDM\#1 scheme, we introduce $\alpha$-OFDM\#3, based on a set $\mathcal{M}$ of $M$ values for $\alpha$ which are constrained by $N_{u}-N \leq$ $\alpha / 2 \pi \leq N-N_{u}$.

The introduction of this particular scheme is relevant as the basis for some practical applications studied in Section 5 . The capacity $C_{\# 3}$ is identical to (28) with the additional constraint on the set of rotations $\mathcal{M}$. Therefore, $C_{\# 3} \leq C_{\# 1}$. This upper-band on the capacity $C_{\# 3}$ is needed for further analysis in the following.

3.3. Capacity Gain. The effect of the $\alpha$-OFDM\#k schemes is to successively slide the OFDM DFT window by different frequency shifts. This generates channel diversity that is highly demanded in outage scenarios. The following lemma shows that the (per subcarrier) fixed channel capacity limit of $\alpha$-OFDM\#1, for any $N_{u}<N$, equals the (per subcarrier) fixed channel capacity of an OFDM system, for $N_{u}=N$. The ratio between the total $\alpha$-OFDM\#1 capacity and the 
total OFDM capacity is then $N_{u} / N$ for a proper choice of $\mathcal{M}$. In contrast, this ratio is on average less than $N_{u} / N$ when a single value for $\alpha$ is used (or similarly when a pure OFDM scheme with $N_{u}<N$ subcarriers is used). This claim is the most important result of this paper and is summarized in the following lemma.

Lemma 1. Consider a single user OFDM system with $N_{u}$ being useful subcarriers and $N$ being total subcarriers without CSIT. Apply $\alpha$-OFDM\#1 with a pattern $\mathcal{M}$ of cardinal $M$ defined in (27) with the constraint $N \mid\left\{M \cdot \operatorname{gcd}\left(N, N_{u}\right)\right\}, \operatorname{gcd}(x, y)$ being the greater common divider of $x$ and $y$. Assuming that the channel coherence time is more than $M$ times the OFDM symbol duration, the $\alpha$-OFDM\#1 capacity $C_{\# 1}$ satisfies

$$
C_{\# 1}=C
$$

with

$$
C=\frac{1}{N} \sum_{m=0}^{N-1} \log \left(1+\frac{\left|H_{0}(m)\right|^{2}}{\sigma^{2}}\right)
$$

being the capacity of the equivalent OFDM system under the fixed channel $\mathbf{H}_{0}$.

Proof. Recall that the $\alpha$-OFDM\#1 capacity reads

$$
C_{\# 1}=\frac{1}{M N_{u}} \sum_{m=0}^{N_{u}-1} \sum_{\alpha \in \mathcal{M}} \log \left(1+\frac{\left|H_{\alpha}(m)\right|^{2}}{\sigma^{2}}\right) .
$$

Since all $\alpha \in \mathcal{M}$ are multiple of $2 \pi$, the frequency shifts correspond to subcarrier jumps. Therefore, after $M$ symbols, every subcarrier indexed by $i \in\{1, \ldots, N\}$ has been used an integer number of times $\lambda_{i} \leq M$. This allows to rewrite $C_{\# 1}$ :

$$
C_{\# 1}=\frac{1}{M N_{u}} \sum_{m=0}^{N-1} \lambda_{m} \log \left(1+\frac{\left|H_{0}(m)\right|^{2}}{\sigma^{2}}\right)
$$

with $\sum_{m=0}^{N-1} \lambda_{m}=M N_{u}$. The variable change $\beta_{m}=N \lambda_{m} / M N_{u}$ leads to

$$
\begin{aligned}
C_{\# 1} & =\frac{1}{N} \sum_{m=0}^{N-1} \beta_{m} \log \left(1+\frac{\left|H_{0}(m)\right|^{2}}{\sigma^{2}}\right) \\
& \leq \frac{1}{N} \log \left(\sum_{m=0}^{N-1} \beta_{m}\left(1+\frac{\left|H_{0}(m)\right|^{2}}{\sigma^{2}}\right)\right) \\
& =\frac{1}{N} \log \left(N+\sum_{m=0}^{N-1} \beta_{m} \frac{\left|H_{0}(m)\right|^{2}}{\sigma^{2}}\right),
\end{aligned}
$$

where the inequality (37) stems from the concavity of the log function.

Without CSIT, one has to assume equal gains at all subcarriers. The best $\beta_{m}$ allocation strategy requires then to maximize all $\beta_{m}$ with sum constraint $\sum_{m} \beta_{m}=N$. This leads to forall $m \in[0, N-1], \beta_{m}=1$. This is the equality case of (37) which is achievable when $N \mid\left\{M \cdot G C D\left(N, N_{u}\right)\right\}$.
Note that this proof cannot be adapted to the $\alpha$-OFDM\#3 scheme for the $\beta_{m}$ 's are restricted by the constraint on $\mathcal{M}$. This means that the achievable outage capacity for $\alpha$ OFDM\#3 is less than the outage capacity for $\alpha$-OFDM\#1; Lemma 1 provides an upper bound on the achievable capacity for $\alpha$-OFDM\#3. As a consequence, if an OFDM system is constrained to use $N_{u}$ consecutive subcarriers with sidebands, using $\alpha$-OFDM allows to reach at most the same outage spectral efficiency as the classical OFDM scheme using all $N$ subcarriers, which, on average, is not achievable with classical OFDM over a fixed set of $N_{u}$ subcarriers. In the following section, simulations are carried out for different scenarios to analyze the potential outage gains of $\alpha$-OFDMbased schemes, before practical applications are discussed. It will in particular be observed that the upper-bound on $C_{\# 3}$ is actually very tight in nondegenerated scenarios.

In terms of performance in block fading channels, the consequence of Lemma 1 is that the outage capacity of $\alpha$ OFDM\#1 equals the outage capacity of the classical OFDM scheme, if it were assumed that all subcarriers were useful ones (i.e., $N_{u}=N$ ). The close behaviour of $\alpha$-OFDM\#3 compared to $\alpha$-OFDM\#1 implies that this scheme also achieves outage capacity performances close to that of the OFDM modulation with all subcarriers in use. On the contrary, when classical OFDM is used on a restricted set $N_{u}<N$, the outage channels may lead to tremendous losses in outage capacity performance; in particular, if the channel coherence bandwidth is close to $N_{u}$ subcarrier spacings, then the outage scenarios correspond to deep fades in the exact position of the $N_{u}$ subcarriers, which entails considerable performance loss.

\section{Simulation and Results}

The 3GPP-LTE OFDM standard is considered in most simulations. We present results for the $1.4 \mathrm{MHz}$ bandwidth $\left(N_{u}=\right.$ $76, N=128)$ and the $10 \mathrm{MHz}$ bandwidth $\left(N_{u}=602, N=\right.$ 1024). In LTE, the null subcarriers on the bandwidth sides do not correspond to guard bands but arise from oversampling at the receiver; as a result, the $N-N_{u}$ empty subcarriers belong to adjacent users. We study the outage capacity and bit error rate (BER) gain assuming that we were allowed to slide the spectrum over those bands while still sending data on $N_{u}$ consecutive subcarriers. This seemingly awkward assumption will prove necessary for the concrete applications in Section 5, in which all users will be allowed to jointly slide their useful bandwidths; this will turn the adjacent illegal spectrum into dynamically usable frequencies. Channels are modeled either as exponential decaying with mean zero and unit variance or as LTE standardized channels [13] with the following characteristics:

(i) extended pedestrian A model (EPA), with RMS delay spread being 43 nanoseconds,

(ii) extended vehicular A model (EVA), with RMS delay spread being 357 nanoseconds,

(iii) extended typical urban model (ETU), with RMS delay spread being 991 nanoseconds. 


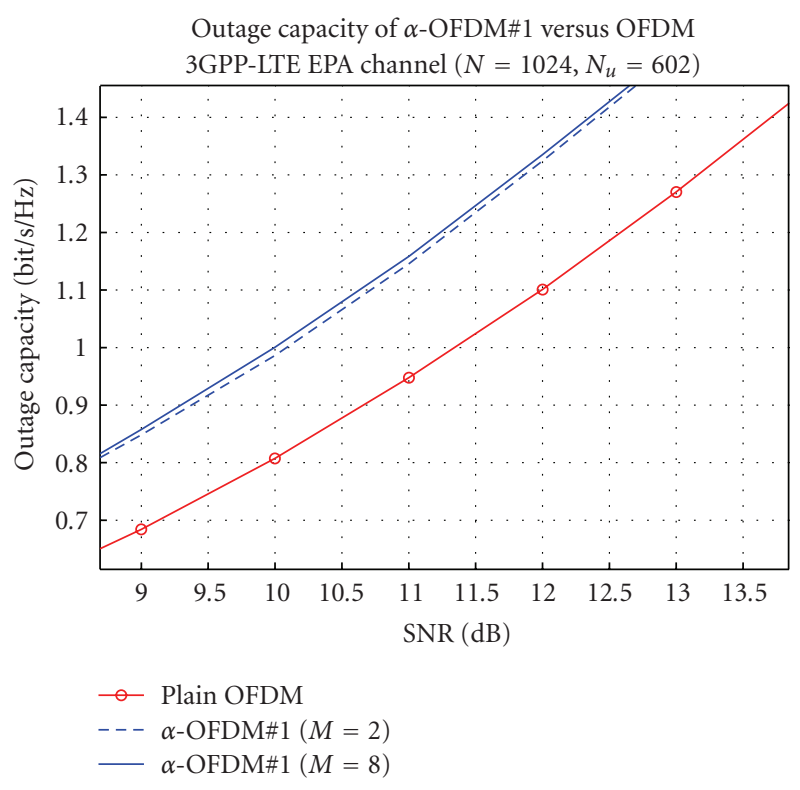

Figure 3: $\alpha$-OFDM\#1 Outage Capacity in 3GPP-LTE EPA.

Figure 3 compares the outage capacity gain of $\alpha$ OFDM\#1 against OFDM in LTE EPA channels for a set $\mathcal{M}$ of length $M=2$ and $M=8$. The bandwidth is 1.4 MHz. A strong SNR gain is provided by $\alpha$-OFDM\# 1 already for $M=2(+1.1 \mathrm{~dB})$, while growing $M$ does not bring significant improvement. This is explained by the fact that $M=2$ suffices to transmit over all available subcarriers, providing already a high diversity gain (however, since a part of the subcarriers is used twice in both transmissions while the others are only used once, the optimal bandwidth usage of the proof in Lemma 1 is not achieved; this requires to transmit as much data on all subcarriers and then demands larger $\mathcal{M}$ sets). Note also that this gain is extremely dependent on the channel length. The shorter the channel delay spread, the more significant the capacity gain.

4.1. Single User with Multiple Antennas. Figure 4 depicts the outage capacity gain of $\alpha$-OFDM schemes versus OFDM in Rayleigh channels using multiple antennas at the transmitter. For $N_{t}$ transmit and receive antennas, the ergodic capacity for the classical OFDM schemes (in plain lines in the figure) reads

$$
C=\frac{1}{N_{u}} \log \operatorname{det}\left(\mathbf{I}_{N_{u}}+\frac{1}{\sigma^{2}} \mathbf{H}_{0}^{\left(N_{u}\right)}\right),
$$

where $\mathbf{H}_{0}^{\left(N_{u}\right)}$ is the $N_{u} \times N_{u}$ matrix extracted from the $N_{u}$ useful rows/columns of $\mathbf{H}_{0}$, while the respective capacity expressions of the $\alpha$-OFDM1 and $\alpha$-OFDM2 schemes are

$$
\begin{gathered}
C_{\# 1}=\frac{1}{M N_{u}} \sum_{\alpha \in \mathcal{M}} \log \operatorname{det}\left(\mathbf{I}_{N_{u}}+\frac{1}{\sigma^{2}} \mathbf{H}_{\alpha}^{\left(N_{u}\right)}\right), \\
C_{\# 2}=\frac{1}{N_{u}} m_{\alpha \in \mathcal{M}} \log \operatorname{det}\left(\mathbf{I}_{N_{u}}+\frac{1}{\sigma^{2}} \mathbf{H}_{\alpha}^{\left(N_{u}\right)}\right),
\end{gathered}
$$

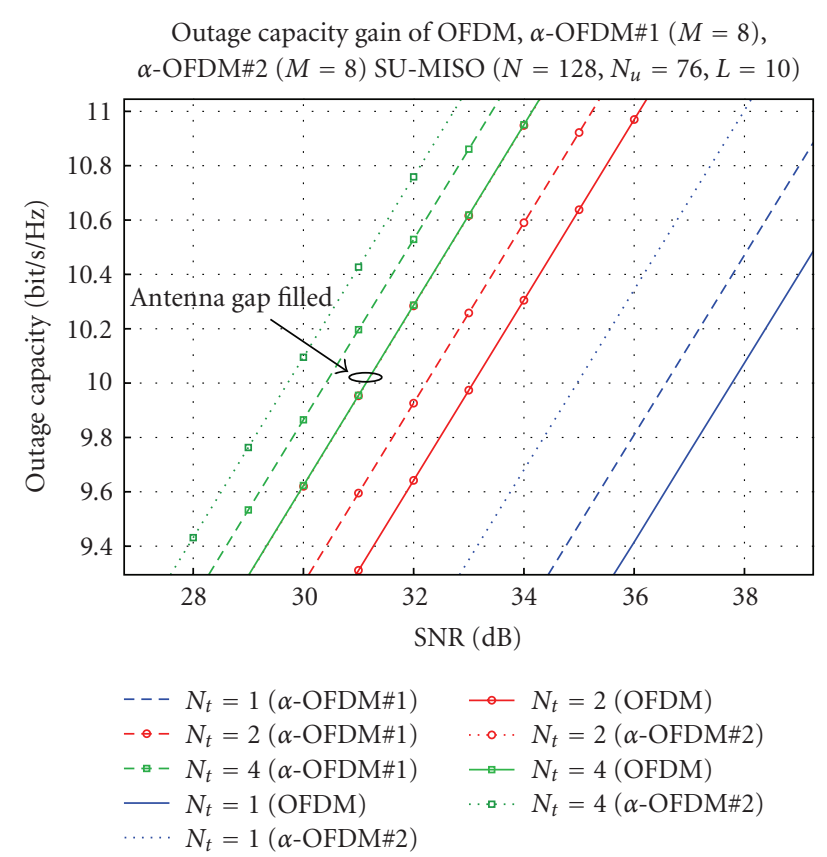

FIgURE 4: Outage capacity gain for $\alpha$-OFDM MISO.

where $\mathbf{H}_{\alpha}^{\left(N_{u}\right)}$ is similarly the $N_{u} \times N_{u}$ matrix taken from the $N_{u}$ useful rows/columns of $\mathbf{H}_{\alpha}$. In the simulations, we use $N_{t}=1,2,4$ transmit and receive antennas.

It is observed that the usage of additional diversity antennas can be partially or fully replaced by $\alpha$-OFDM schemes. Channel diversity from the space domain can then be traded off with diversity in the time-domain thanks to $\alpha$ OFDM, reducing then the cost of extra antennas. However, particular care is demanded to appreciate those results. Indeed, the performance gains in terms of outage capacity and BER are highly dependent on the exact definition of the outage conditions and the channel delay spread. In mobile short-range wireless communications, bursty transmissions using $\alpha$-OFDM can therefore be performed at higher rates.

4.2. Multicell Systems. In multicell systems, intercell interference engenders outage situations whenever the terminal's channel to the base station is in outage and the channels to the interferers in adjacent cells are strong. Using $\alpha$ OFDM in its own cell, not only will the terminal diversify its own channel but it will also face different interference patterns. Therefore, it is even less likely to simultaneously be confronted to bad channel conditions in its own cell and strong interference over $M$ consecutive $\alpha$-OFDM symbols. Figure 5 provides this analysis, in which a receive user faces interference from a single adjacent cell at varying signal-tointerference ratios (SIRs). The SNR is fixed to SNR $=15 \mathrm{~dB}$. The channel lengths at both user's cell and the interfering cell are set to $L=3$ while $N_{u}=76$ and the DFT size is $N=128$. At high SIR, one finds again the $\alpha$-OFDM capacity gain observed in Figure 3. Around SIR $=20 \mathrm{~dB}$, a level for which interference becomes a relevant factor, the outage gain due to $\alpha$-OFDM\#1 is more than $3 \mathrm{~dB}$, which is twice the capacity gain obtained in single-cell OFDM. As discussed 


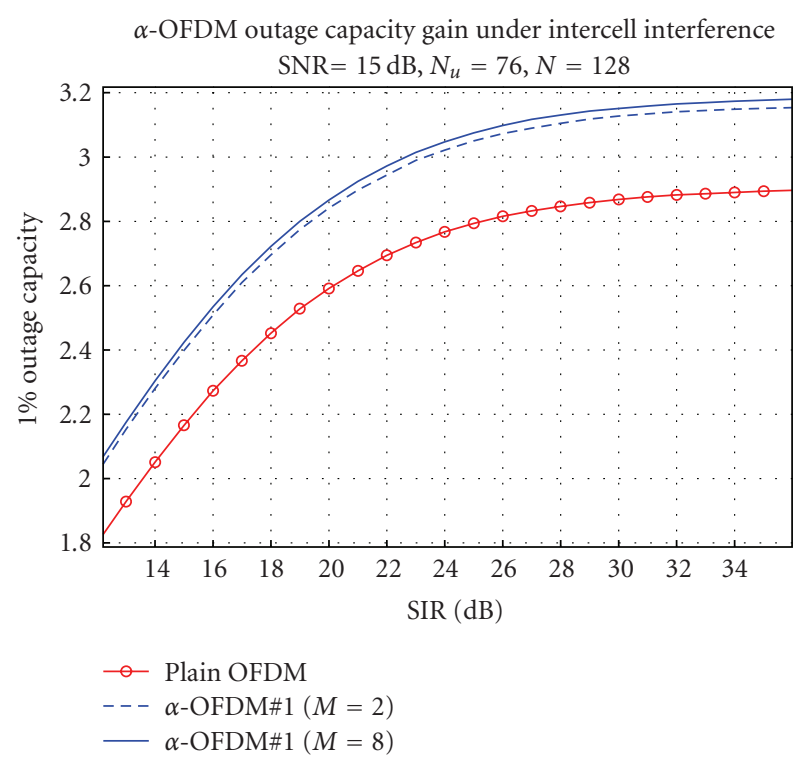

FIGURE 5: $\alpha$-OFDM\#1 with intercell interference.

in the previous section, those gains are even larger if the considered outage is less than $1 \%$ but are less important for more frequency selective channels.

\section{Applications}

In this study, on the specific example of the $1.4 \mathrm{MHz}$ LTE frequency band, we assumed that one was allowed to transmit data on side-bands to capitalize on channel diversity. This requires that those bands are not in use. In the following we propose schemes for service providers to overcome this problem by sacrificing a small part of the total bandwidth.

5.1. $\alpha$-LTE. In the LTE context, service providers are allowed to use up to $20 \mathrm{MHz}$ bandwidth that they can freely subdivide in several chunks. Consider that one decides to split the available bandwidth in 16 chunks of $1.25 \mathrm{MHz}$ each. Those chunks are composed of 76 subcarriers which are oversampled to 128 for ease of computation at the transmitter and at the receiver. We propose to sacrifice 4 subcarriers per chunk that then results in $4 \times 16=64$ free subcarriers in total. Those 64 subcarriers are gathered in two subbands of 32 subcarriers, placed on both sides of the $20 \mathrm{MHz}$ band. By synchronously using $\alpha$-OFDM on every chunk for a maximum of 16 users, we can design a system of $N_{u}=76-4=72$ effective subcarriers per user over a total $N=72+64=136$ available subcarriers. Indeed, the 64 spared subcarriers are reusable to all 16 users by sliding their individual DFT windows.

Figure 7 illustrates a simplified version of the aforedescribed scheme, with 4 chunks instead of 16 . In this particular example, an $M=3 \alpha$-OFDM-based scheme is used that synchronously exploits the left subcarriers, of the dotted part of each chunk, then the central subcarriers and finally the right subcarriers in any three consecutive OFDM symbols

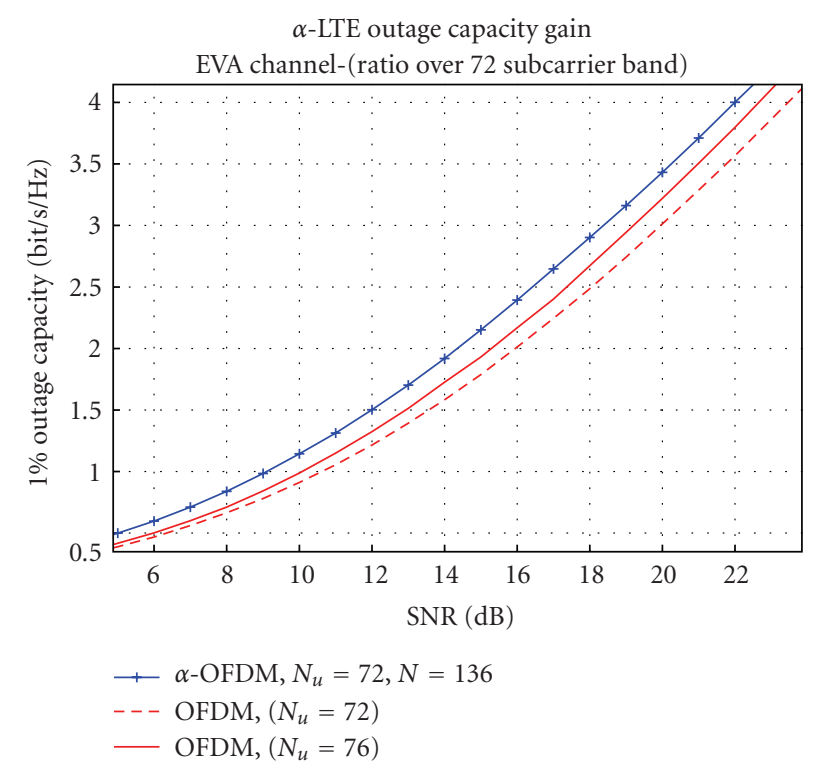

FIGURE 6: LTE and $\alpha$-LTE outage capacity.
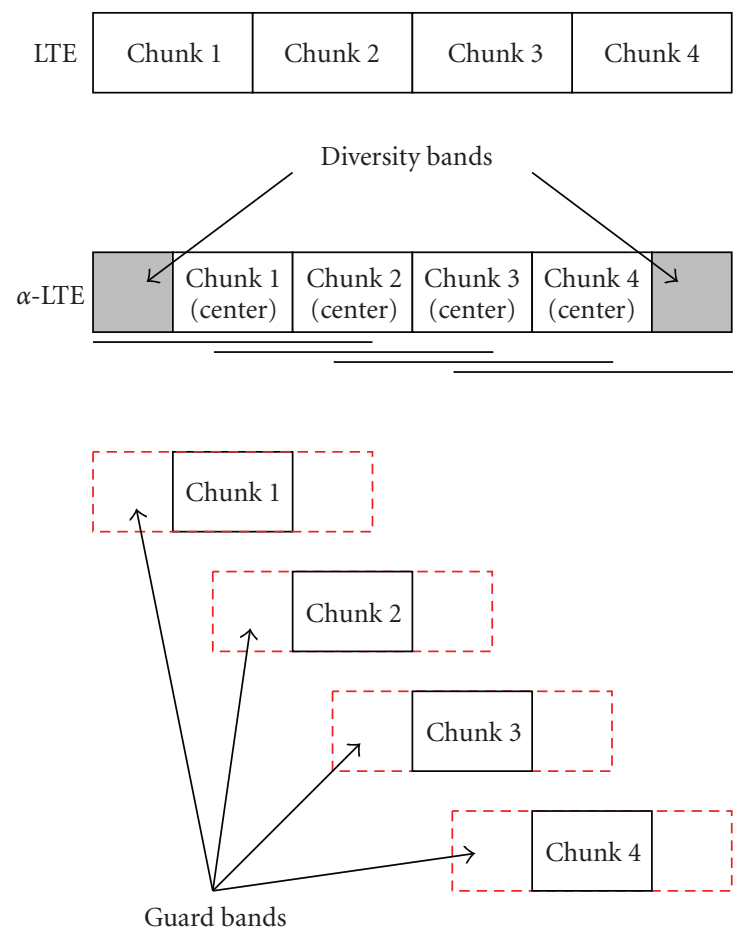

FIgURE 7: $\alpha$-LTE.

$\left(\mathbf{s}^{(3 k)}, \mathbf{s}^{(3 k+1)}, \mathbf{s}^{(3 k+2)}\right)$. Therefore, data will always be sent on nonoverlapping bands. In our particular example, we use a 136-DFT for a signal occupying the central 72-subcarrier band.

The gain of $\alpha$-LTE lies in outage BER and also, at a low-to-medium SNR, in outage capacity. Indeed, the lack of 4 subcarriers induces a factor $72 / 76$ on the total outage capacity $C$ which scales like $N_{u} \log (\mathrm{SNR})$ at high SNR. But at low-to-medium SNR, the gain discussed in Section 3 appears 
and overtakes the loss in outage capacity introduced by the factor $72 / 76$.

Figure 6 provides the simulation results obtained in $1 \%$ outage capacity for a transmission through 3GPP-EVA channels with $N_{u}=72, N=136$ in the low-to-medium SNR regime. For fair comparison, we plotted the outage capacity cumulated over a bandwidth of 76 subcarriers (therefore, when $N_{u}=72,4$ subcarriers are left unused) that we normalize by 76 . At low-to-medium SNR, a gain in capacity is observed, despite the loss of 4 subcarriers, while at high SNR the classical OFDM fills the gap with our improved method.

5.2. Ultra-Wideband. 3GPP LTE is not the only standard to allow its allocated bandwidth to be divided into many OFDM systems. For instance, UWB systems, that cannot manage very large DFT computations, divide their allocated bandwidth into multiple OFDM chunks. Such a scheme is commonly referred to as multicarrier OFDM. $\alpha$-LTE can be generalized to such systems of total bandwidth $W$ with $N$ subcarriers, subdivided into $K$ subbands. In classical OFDM, this results in chunks of size $N / K$ and therefore, without oversampling, to a DFT size $N / K$.

With $\alpha$-OFDM, one can introduce a guard band of $G$ subcarriers, to result into $K$ chunks of size $(N-G) / K$ and to an $((N-G) / K+G)$-DFT. By making $(N, K)$ grow large with a constant ratio, the DFT size tends to $N / K+G$, while the number of useful subcarriers is fixed to $N / K$.

The loss in outage capacity per chunk at high SNR is then fairly reduced while the gain in outage BER per chunk is kept constant independently of $(N, K)$. In this asymptotic scenario, $\alpha$-OFDM does not require to sacrifice any frequency band but provides diversity and outage capacity gain.

5.3. Cognitive Radios in Unlicensed Bands. Advanced techniques of channel sensing allow cognitive radios [9] to figure out the spectral occupation of the neighbouring frequencies. For bursty systems, it could be convenient to reuse these free bandwidths $\mathcal{W}_{e}$. By increasing the rotation pattern $\mathcal{M}$ accordingly, for instance, $\mathcal{M}=\left\{0,2 \pi N \mathcal{W}_{e} / \mathcal{W}\right\}$, it is possible to dynamically gain in channel diversity. The terminal can be informed of the communication mode, that is, of the set $\mathcal{M}$, to be used in a few bits through a dedicated control channel. This especially allows for software-defined dynamic spectrum allocation in an OFDM network, whether outage capacity or other performance metric is sought for.

\section{Conclusion}

In this paper, flexible OFDM schemes based on the $\alpha$-OFDM concept are proposed. $\alpha$-OFDM allows to exploit large bandwidths to obtain outage gains for bursty OFDM systems. $\alpha$-OFDM requires a minor change compared to OFDM which offers no capacity improvement in its raw form. Nevertheless, $\alpha$-OFDM provides a way to exploit reusable frequency bands and shows outage capacity improvement compared to the classical OFDM modulation. In multicell scenarios, $\alpha$-OFDM can be exploited to mitigate intercell interference. Also schemes based on $\alpha$-OFDM can be used to efficiently replace extra antennas at the transmitter. A large set of applications is derived from $\alpha$-OFDM, such as $\alpha$-LTE, a proposed evolution of the LTE standard which shows performance gain in packet-switched mode and short channel delay spread. $\alpha$-OFDM coupled to channel sensing methods also enters the scope of software-defined radios as it allows to smartly exploit the available bandwidth.

\section{Acknowledgment}

This work was partially supported by the European Commission in the framework of the FP7 Network of Excellence in Wireless Communications NEWCOM++.

\section{References}

[1] ANSI/IEEE Std 802.11, 1999 Edition (R2003), http:// standards.ieee.org/getieee802/download/802.11-1999.pdf.

[2] "Air Interface for Fixed and Mobile BroadbandWireless Access Systems," http://standards.ieee.org/getieee802/download/802 .16e-2005.pdf.

[3] S. Sesia, I. Toufik, and M. Baker, "LTE, The UMTS Long Term Evolution: From Theory to Practice," Wiley and Sons, 2009.

[4] J. A. C. Bingham, "Multicarrier modulation for data transmission: an idea whose time has come," IEEE Communications Magazine, vol. 28, no. 5, pp. 5-14, 1990.

[5] E. Biglieri, J. Proakis, and S. Shamai, "Fading channels: information-theoretic and communications aspects," IEEE Transactions on Information Theory, vol. 44, no. 6, pp. 26192692, 1998.

[6] P. Viswanath, D. N. C. Tse, and R. Laroia, "Opportunistic beamforming using dumb antennas," in Proceedings of the IEEE International Symposium on Information Theory, p. 449, Lausanne, France, June 2002.

[7] R. Müller, R. Bains, and J. Aas, "Compact MIMO receive antennas," in Proceedings of the 43rd Annual Allerton Conference on Communications, Control and Computing, Monticello, Ill, USA, September 2005.

[8] A. Dammann and S. Kaiser, "Performance of low complex antenna diversity techniques for mobile OFDM systems," in Proceedings of the 3rd International Workshop on Multi-Carrier Spread-Spectrum \& Related Topics (MC-SS '01), pp. 53-64, Oberpfaffenhofen, Germany, September 2001.

[9] J. Mitola III and G. Q. Maguire Jr., "Cognitive radio: making software radios more personal," IEEE Personal Communications, vol. 6, no. 4, pp. 13-18, 1999.

[10] S. Haykin, "Cognitive radio: brain-empowered wireless communications," IEEE Journal on Selected Areas in Communications, vol. 23, no. 2, pp. 201-220, 2005.

[11] R. M. Gray, Toeplitz and Circulant Matrices: A Review, Now, Norwell, Mass, USS, 1977.

[12] C. E. Shannon, "A mathematical theory of communication," The Bell System Technical Journal, vol. 27, pp. 379-423, 623656, 1948.

[13] Ericsson, Nokia, Motorola, Rohde, and Schwarz, "Proposal for LTE channel models," TSG-RAN WG4 Meeting \#43 R4070572, Kobe, Japan, May 2007. 